Noname manuscript No.

(will be inserted by the editor)

\title{
A note on the set-up under the Draupner wave
}

2 Thomas A.A. Adcock

4 Received: date / Accepted: date

5 Abstract The Draupner wave is the most famous 'freak' or 'rogue' wave obser-

6 vation. In this note we examine the short-term probability of this event in both

7 following and crossing sea-states. We run random simulations and generate both

8 second- and approximate third-order statistics. The wave is found to be more

9 probable in a following sea. The measured waves showed both a set-up (under the

10 giant wave) as well as set-downs under other large waves in the record - this is

11 found to be consistent with a crossing sea.

12 Keywords Draupner wave $\cdot$ New Year Wave Ocean crest statistics

\section{Introduction}

${ }_{14}$ The 'Draupner' wave, or 'New Year Wave', is the most famous measurement of a rogue wave in the ocean (see review papers on rogue waves Kharif and Pelinovsky

16 (2003); Dysthe et al (2008); Adcock and Taylor (2014)). The wave was recorded 17 by a downward pointing laser on the Draupner platform in the North Sea. Figure 181 shows the free surface record with the crest reaching $18.5 \mathrm{~m}$ in a sea-state of ${ }_{19} H_{s}=12 \mathrm{~m}$. Minor damage beneath the platform confirms a very large wave did occur. The measurement of the wave is described in detail by Haver (2004).

T.A.A. Adcock

Department of Engineering Science

University of Oxford 


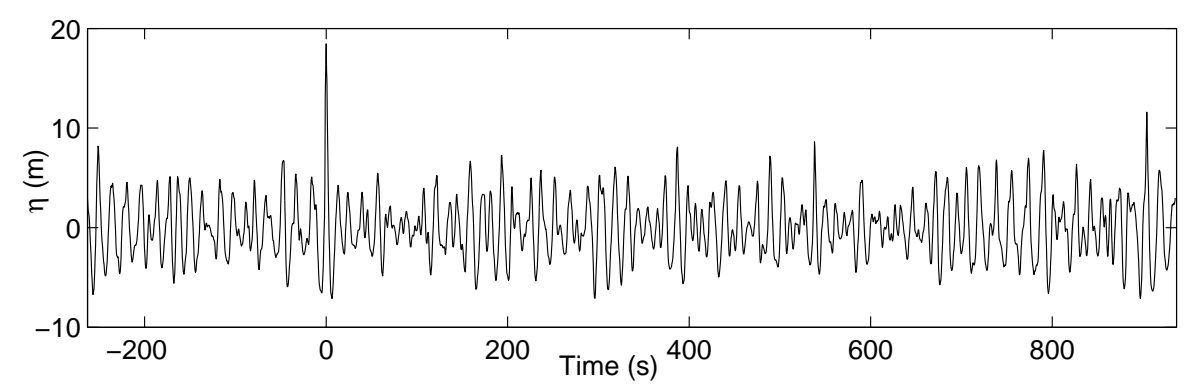

Fig. 1 The measured free surface around the Draupner wave. The Draupner wave is at Time $=0$.

There has been considerable interest in the physics and statistics of the Draupner wave. A common suggestion is that the non-linear Benjamin-Feir instability played a part in the formation (Chabchoub et al, 2010; Didenkulova et al, 2013; Janssen, 2015). However, the water depth at the platform was $70 \mathrm{~m}$ (giving $k d \sim 1.6$ ) which would almost completely inhibit the Benjamin-Feir instability (as would the broad-banded spectrum and short-crestedness of the sea-state). A recent paper by Fedele et al (2016) carried out an elegant study of the Draupner sea-state using a higher order spectral scheme and concluded that there was no evidence that non-linear instabilities played a part in the formation of the Draupner wave. Whilst it is, of course, possible to make waves which look like Draupner using 'breather' type solutions, this does not imply in itself that the more straightforward suggestion of superposition of random linear waves is not the right one.

The Draupner wave does, however, exhibit some unusual physical properties. One is that, if all the energy were going in one main direction (a 'following' sea) there is some evidence that the wave should have broken. More suggestive, is that under the wave there is a low frequency increase in the water level rather than the expected set-down (Walker et al, 2004). This, and other evidence, led to the suggestion by Adcock et al (2011) that the wave was the result of two wave systems which were crossing (i.e. propagating in very different directions). There is some hindcast evidence for this (e.g. Adcock et al (2011); Cavaleri et al (2016)) although these generally suggest the two wave systems were not separated by as large an angle as Adcock et al. suggest. It is also unclear why the set-up only occurs around 
the giant wave and not around other waves in the twenty minute record where the

44 expected set-down occurred (Adcock and Taylor, 2009).

The statistics of the wave are obviously also of interest. Wave-crest statistics

70 2.1 Linear random waves

are typically broken down into long- and short-term (the statistics of the sea-state and of the individual waves within a given sea-state respectively). Although severe, a $12 \mathrm{~m}$ significant wave-height is not abnormal for a winter storm in the middle of the North Sea. Hindcasting the sea-state has proved not to be straightforward (e.g. Janssen (2015) - the latest careful work by Cavaleri et al (2016) under-estimates the significant wave-height of the sea-state by one metre. This hints that the seastate was rapidly changing. This is suggestive but is not taken further in this note.

This note examines only short-term statistics. The Draupner wave is more of an outlier if short-term statistics are considered. By any calculation a crest more than 1.5 times the significant wave-height is an unusual event in a 20 minute record at a single point. The most commonly used crest distribution is the Forristall distribution. This is derived from a fit to second order numerical simulations of following sea states and would not be expected to give as accurate results as second order simulations of the specific water depth and sea-state. The model is also sensitive to the choice of wave-number used in the calculation. The Forristall distribution would also not be expected to give accurate results in crossing sea-states (Christou et al, 2009). Prevosto and Bouffandeau (2002) carried out a number of simulations based on linear dynamics but with different corrections to the bound harmonics. However, whilst they considered a number of interesting cases, they did not simulate second order difference waves for a realistically spread sea in $70 \mathrm{~m}$ water depth. They also did not consider a crossing sea. This note aims to fill this gap in the literature.

\section{Methods}

We start by generating time-histories of linear random waves. We use an approach slightly adapted from that described in Gōda (2010) taking into account the work 


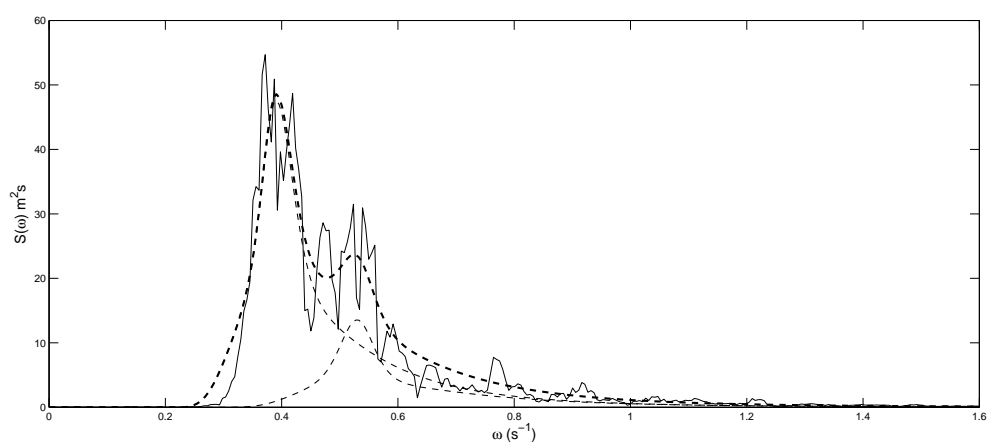

Fig. 2 Measured and simulated power spectra. Solid line - measured spectrum (20 minutes); thin dashed lines - two JONSWAP spectra described in paper; thick dashed line - sum of two dashed JONSWAP spectra.

of Tucker et al (1984). For computational purposes we choose to simulate periods of 22.5 minute sections and extract the 20 minutes in the middle of this (to remove any end effects in the calculation of the higher order terms). We generate enough records to give stable statistics for crest amplitudes up to $18.5 \mathrm{~m}$.

In this study we choose to base our spectrum on the measured data. Figure 2 shows the omni-directional power spectrum measured during the 20 minutes around the giant wave. It appears to be double peaked suggesting that it might be the results of two systems interacting (note that the spectra Prevosto and Bouffandeau (2002) used does not appear to be double peaked). We fit the measured spectrum with two JONSWAP spectra as shown in the figure. The key parameters of these fits are for the main peak $\omega_{p}=0.39 \mathrm{rad} \mathrm{s}^{-1}$ and $\gamma=2.5$ and for the second $\omega_{p}=0.53 \mathrm{rad} \mathrm{s}^{-1}$ and $\gamma=3.3$. The significant wave-heigh of the two seas is $10.5 \mathrm{~m}$ and $5.9 \mathrm{~m}$.

To calculate the higher order harmonics we need to use a directional distribution of energy. We assume that each of the two spectra has the frequency dependent directional distribution of energy about a mean wave directional given by Ewans (1998) based on his analysis of measured data (generally consistent with the findings of Adcock and Taylor (2009) for this sea-state). We consider two cases. In the 'following' case both spectra have the same mean direction. In the 'crossing' case we assume that the two spectra are separated by $120^{\circ}$ as hypothesised by Adcock et al (2011). 
2.2 Second order harmonics

We calculate the second order bound harmonics using the interactions given by Dalzell (1999); Forristall (2000). The general form of the second order free surface, et $a_{2}$ is given by

$$
\eta_{2}=\sum_{n} \sum_{m} a_{n} a_{m} B^{+} \cos \left(\phi_{n}+\phi_{m}\right)+\sum_{n} \sum_{m} a_{n} a_{m} B^{-} \cos \left(\phi_{n}-\phi_{m}\right),
$$

where $a$ are the amplitudes of the interacting components which have phase phi.

The interaction kernels, $B^{+}$and $B^{-}$for the sum and difference terms respectively, are functions of the frequency and direction of the interacting components as well as the water depth. These can be found in the references above. As noted in Adcock and Draper (2015) (see also Janssen (2009)) in calculating the low frequency 'difference' term these break down when the interaction between components with very different frequencies are evaluated (causing significant errors). We therefore set the difference interaction to zero when

$$
\frac{2\left|\omega_{1}-\omega_{2}\right|}{\omega_{1}+\omega_{2}}>0.75
$$

where $\omega_{1}$ and $\omega_{2}$ are the frequencies of the interacting components. Thus the interactions with the tail of the spectrum are excluded from the calculation of the low frequency difference term.

2.3 Third order harmonics

In this note we briefly consider third order bound harmonics. In principle, these could be evaluated from Madsen and Fuhrman (2006). However, this is computationally demanding and so we simplify this following Walker et al (2004). We assume that the bound third order component is given by the 3rd order coefficient of the Stokes expansion (i.e. the uni-directional and narrow bandwidth limit). Thus, following Walker et al., the third order correction is calculated as

$$
\eta_{3}=\frac{1.57}{70^{2}}\left(\eta^{2}-3 \hat{\eta}^{2}\right) \eta
$$

where $\eta$ is the linear free surface and $\hat{\eta}$ is the Hilbert transform of the linear free surface. This estimate for the New Year Storm is based on a finite depth Stokes 
wave. In following seas this is expected to overestimate the magnitude of the third order term (in deep water for a typical storm wave Adcock and Taylor (2016) found this over-predicted the third harmonic by $8 \%$ ). In crossing seas it is likely to be less accurate. Therefore, for our crossing sea simulations we calculate the third harmonics on each of the two systems separately, and assume there is zero third order interaction between the two systems.

\subsection{Other non-linear dynamics}

Bound interactions beyond third order will be small $\left((k a)^{3} \sim 0.035\right.$ where $a$ and $k$ are estimates of the amplitude and characteristic wavenumber of the Draupner wave) and we do not examine these in this note.

As noted in the introduction, there is weak evidence that non-linear dynamics causes significantly more large waves than would be expected in the linear model for this water depth and for broadbanded, directionally-spread seas (we note some evidence to the contrary in the work of Latheef and Swan (2013)). The results of Janssen (2015) do suggest that for this sea-state some non-linear focussing is possible although we do not take this further here. However, there is evidence that non-linear physics can lead to a reduction in the directional spread under large waves in comparable water depths (Swan and Latheef, 2016). At least in deep water, there is some evidence that extreme waves would move to the front of wave-packets Adcock et al (2015). Both of these non-linear changes to the shape of extreme waves would have an influence on the bound waves - however this physics is not modelled in the present paper. We also do not attempt to model wave breaking.

\section{Results}

Figure 3 presents the key results from our numerical simulations. The figure shows the predicted probabilities of the largest crest in an $11.97 \mathrm{~m}$ seas-state in a twenty minute period. The figure shows that the probability of a given extreme crest height being exceeded is much larger in the following sea than in the crossing sea 


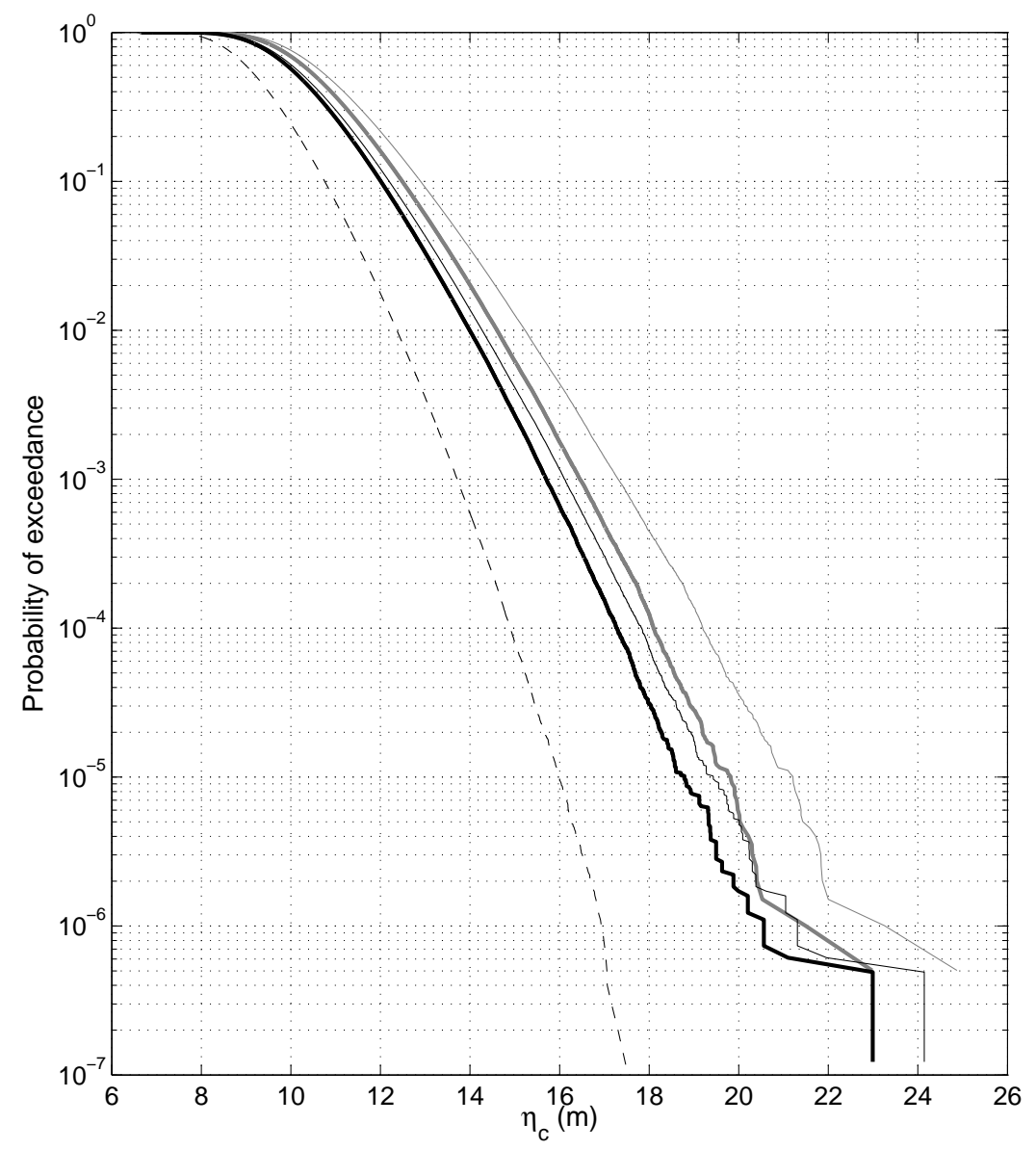

Fig. 3 Probabilities of exceedance for the largest crest in 20 minute simulation. Black crossing sea; grey - following sea. Thick line - second order simulation; thin line - third order simulation. Dashed line - linear.

(consistent with Christou et al (2009)). The approximate inclusion of third order harmonics clearly makes a significant difference to the exceedance probabilities.

Table 1 compares the probability of a single random crest greater or equal to that of Draupner with different models. The numbers are comparable with the estimates presented in Prevosto and Bouffandeau (2002). The differences in the exceedance probabilities calculated here (as with those in Prevosto and Bouffan- 


\begin{tabular}{ll}
\hline Model & $P\left(\eta_{c}>18.5\right)$ \\
\hline 2nd order following & $5.7 \times 10^{-7}$ \\
2nd order crossing & $1.5 \times 10^{-7}$ \\
3rd order following & $2.7 \times 10^{-6}$ \\
3rd order crossing & $3.6 \times 10^{-7}$ \\
\hline
\end{tabular}

Table 1 Probability of a single crest exceeding $18.5 \mathrm{~m}$.

deau (2002)) show significant sensitivity to the modelling choices and assumed sea-state - this is of practical concern to the engineer.

As well as the probability of the extreme crest there are some other interesting features in the field data which can be examined. One feature of note is that the second largest wave in the twenty minutes has a crest of $11.59 \mathrm{~m}$. In this sea-state this is a relatively large wave although it falls slightly below what would usually be described as a freak or rogue wave. It is interesting to examine the chances of this wave as well as that of the more famous crest recorded fifteen minutes earlier. In the third order following-sea simulations (the one with the highest probabilities), we would expect a wave this large in approximately $30 \%$ of 20 minute records. Although this does not make a major change to the chances of observing this time-history, the joint probability of the main wave and the second largest wave is significantly less than the probability of just the giant wave. This makes this sea-state still more interesting.

It was noted in the introduction that an unexplained feature of the crossing sea state theory of Adcock et al (2011) was that the set-up under the giant wave was not observed under other large waves (which showed a set-down). Examination of the records in this study shows that in crossing sea-states it is possible to get either a set-up or a set-down depending on which combination of waves happens to be in phase during the extreme crest. To demonstrate this Figure 4 shows the magnitude of the second order difference terms under extreme crests in crossing seas. There is significant variation (as was found in following seas by (Adcock et al, 2015)). Plainly, if the Draupner sea-state was crossing event a set-up under the giant crest with smaller waves showing a set-down is not implausible.

We note that in the following sea simulations all crests greater than about 12 $m$ have a set-down under them. 


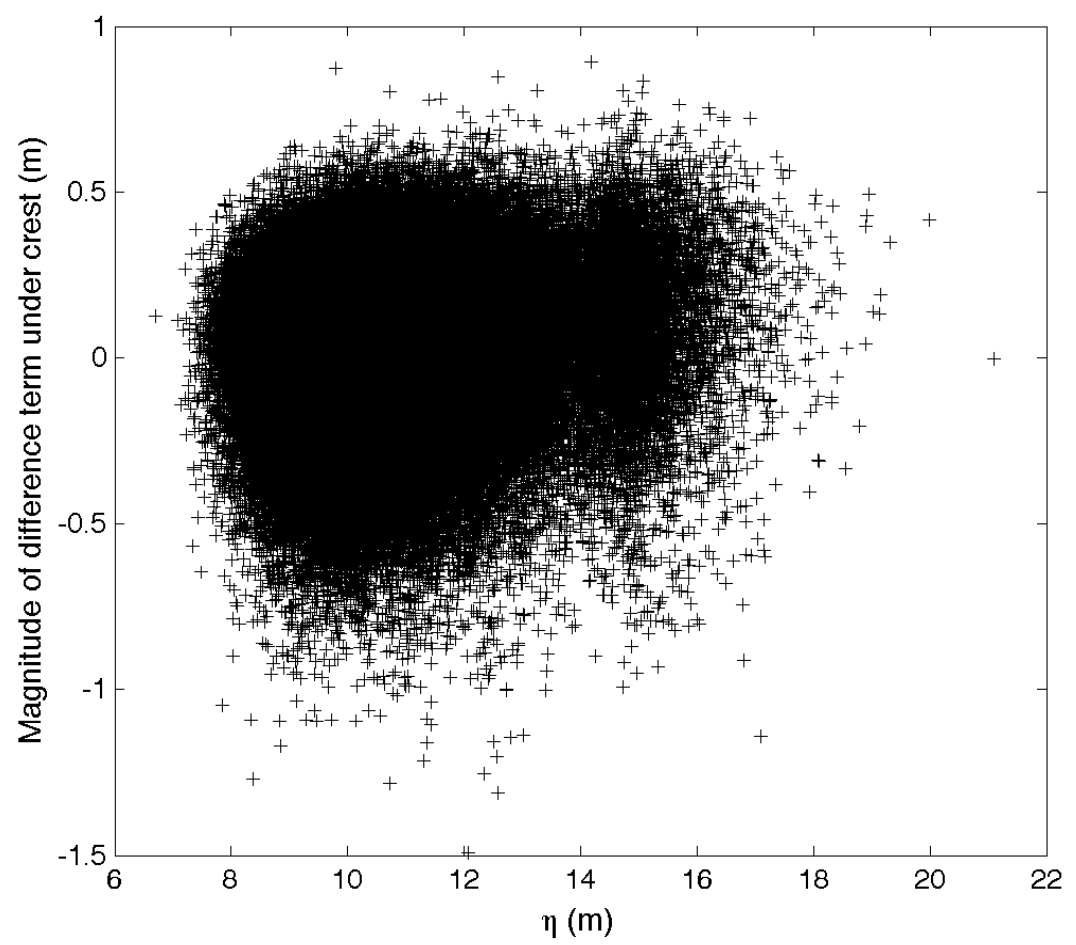

Fig. 4 Magnitude of the low frequency set-up/set-down under large crest in crossing seas. Crest on $\mathrm{x}$-axis is to 2nd order. Only a subset of the data was used in this plot for clarity.

\section{Discussion}

The probabilities of exceedances calculated herein are consistent with expectations.

Perhaps of most interest is whether these results support or contradict the theory that the Draupner wave occurred in a crossing sea. On the one hand, the results presented here suggest that the Draupner wave more likely to occur in a following sea than in a crossing one. Given that the wave was observed, this perhaps reduces the likelihood of the crossing sea theory being correct. Set against this, we have resolved one of the key problems with this crossing sea theory - namely that other waves in the record showed a set-down - these simulations show that this would not be unexpected in a crossing sea.

These simulations highlight the importance of including third-order harmonics in wave-crest estimates (and hence 'air-gap' calculations) in steep sea-states. As 


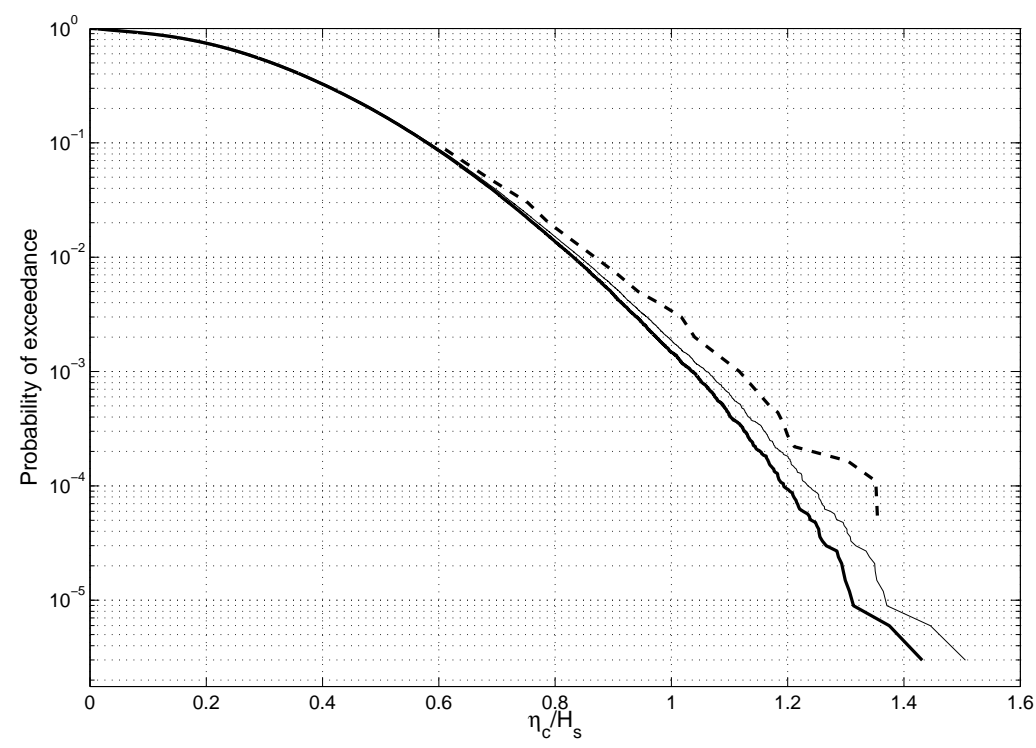

Fig. 5 Comparison of second and third order simulations of crest height, $\eta_{c}$, with laboratory experiments. Black dashed - Imperial College experiments (see Latheef \& Swan (2013)); thin solid line - third order simulations; thick solid line - second order simulations.

a different example, we consider the measurements made in the laboratory study by Latheef and Swan (2013). We choose to examine the case shown in Figure 8(d) of their paper (which shows good agreement with experiments from a different laboratory). This case has $\frac{1}{2} H_{s} k=0.122$ and has $k d=2.019$. The extreme waves measured in this case are significantly under-estimated by the Forristall distribution. Figure 5 shows our second order simulation results using an underlying spectrum identical to that used in the experiments (which are close to the Forristall distribution in this case). The third-order simulations are significantly closer to the measurements - however, even this underestimates the crests of the larger waves.

This note, as with previous papers, shows that the Draupner wave is an improbable event in a linear + bound harmonic model. However, it is by no means impossible. Let us say that there are 10 separate wave sensors in the North Sea, and three severe storms every year with 3000 waves (following Tromans and Vanderschuren (1995)). Most storms will be following seas (see for instance Rodriguez 
et al (2002)) so let us conservatively use the second order probability calculated in the paper of $5.7 \times 10^{-7}$ (although of course different locations will have different depths and storms have different steepnesses). Based on these very crude assumptions we would expect to observe a Draupner like wave once every 20 or so years. Set against this, it is difficult to calculate the chances of a non-linear breather forming in the open ocean - partly because it is almost impossible to see how a breather could arise in a broadbanded directionally spread sea.

\section{Conclusions}

In this study we have carried out second and (approximate) third order calculations of wave crest statistics using the spectrum measured during the Draupner wave. Our estimates for the probability of this wave show some variability depending on the model chosen but are in line with previous estimates. A crest of the height measured at the Draupner platform is much more likely in a following sea than in a crossing sea. Our simulations show that in crossing seas, some large waves can have a set-up whilst others can have a set-down, consistent with what was measured around the Draupner wave.

Acknowledgements I thank Prof Paul Taylor for his help with this note.

\section{References}

Adcock TAA, Draper S (2015) The Second Order Contribution to Wave Crest Amplitude - Random Simulations and New Wave. In: The Twenty-fifth International Offshore and Polar Engineering Conference, International Society of Offshore and Polar Engineers

Adcock TAA, Taylor PH (2009) Estimating ocean wave directional spreading from an Eulerian surface elevation time history. Proceedings of the Royal Society of London A: Mathematical, Physical and Engineering Sciences 465(2111):33613381

Adcock TAA, Taylor PH (2014) The physics of anomalous ("rogue") ocean waves. Reports on Progress in Physics 77(10):105,901 
Adcock TAA, Taylor PH (2016) Fast and local non-linear evolution of steep wavegroups on deep water: A comparison of approximate models to fully non-linear simulations. Physics of Fluids (1994-present) 28(1):016,601

Adcock TAA, Taylor PH, Yan S, Ma QW, Janssen PAEM (2011) Did the Draupner wave occur in a crossing sea? Proceedings of the Royal Society of London A: Mathematical, Physical and Engineering Sciences 467(2134):3004-3021

Adcock TAA, Taylor PH, Draper S (2015) Nonlinear dynamics of wave-groups in random seas: unexpected walls of water in the open ocean. Proc R Soc A 471(2184):20150,660

Cavaleri L, Barbaroil F, Benetazzo L A Bertotti, Bidlot JR, Janssen P, Wedi N (2016) The Draupner wave: a fresh look and the emerging view. in press, Bulletin of the American Meteorological Society

Chabchoub A, Vitanov N, Hoffmann N (2010) Experimental evidence for breather type dynamics in freak waves. PAMM 10(1):495-496

Christou M, Tromans P, Vanderschuren L, Ewans K (2009) Second-order crest statistics of realistic sea states. In: Proc. of the 11th Int. Workshop on Wave Hindcasting and Forecasting, Halifax, Canada, pp 18-23

Dalzell JF (1999) A note on finite depth second-order wave-wave interactions. Applied Ocean Research 21(3):105-111

Didenkulova II, Nikolkina IF, Pelinovsky EN (2013) Rogue waves in the basin of intermediate depth and the possibility of their formation due to the modulational instability. JETP letters 97(4):194-198

Dysthe K, Krogstad HE, Müller P (2008) Oceanic rogue waves. Annu Rev Fluid Mech 40:287-310

Ewans KC (1998) Observations of the directional spectrum of fetch-limited waves. Journal of Physical Oceanography 28(3):495-512

Fedele F, Brennan J, De León SP, Dudley J, Dias F (2016) Real world ocean rogue waves explained without the modulational instability. Scientific Reports 6

Forristall GZ (2000) Wave crest distributions: Observations and second-order theory. Journal of Physical Oceanography 30(8):1931-1943

Gōda Y (2010) Random seas and design of maritime structures. World scientific Haver S (2004) A possible freak wave event measured at the Draupner jacket January 1 1995. Rogue waves 2004 pp 1-8 
Janssen PAEM (2009) On some consequences of the canonical transformation in the hamiltonian theory of water waves. Journal of Fluid Mechanics 637:1-44

Janssen PAEM (2015) How rare is the Draupner wave event. Tech. Rep. 775, European Centre for Medium-Range Weather Forecasts

Kharif C, Pelinovsky E (2003) Physical mechanisms of the rogue wave phenomenon. European Journal of Mechanics-B/Fluids 22(6):603-634

Latheef M, Swan C (2013) A laboratory study of wave crest statistics and the role of directional spreading. Proc R Soc A 469(2152):20120,696

Madsen PA, Fuhrman DR (2006) Third-order theory for bichromatic bi-directional water waves. Journal of Fluid Mechanics 557:369-397

Prevosto M, Bouffandeau B (2002) Probability of occurrence of a "giant" wave crest. In: ASME 2002 21st International Conference on Offshore Mechanics and Arctic Engineering, American Society of Mechanical Engineers, pp 483-490

Rodriguez G, Soares CG, Pacheco M, Pérez-Martell E (2002) Wave height distribution in mixed sea states. Journal of Offshore Mechanics and Arctic Engineering 124(1):34-40

Swan C, Latheef M (2016) Extreme surface water waves in realistic directionally spread seas. In: Extreme events and rogue waves seminar, Bad Honnef, Germany

Tromans PS, Vanderschuren L (1995) Response based design conditions in the North Sea: Application of a new method. In: Offshore Technology Conference, Offshore Technology Conference

Tucker MJ, Challenor PG, Carter DJT (1984) Numerical simulation of a random sea: a common error and its effect upon wave group statistics. Applied Ocean Research 6(2):118-122

Walker DAG, Taylor PH, Eatock Taylor R (2004) The shape of large surface waves on the open sea and the Draupner New Year wave. Applied Ocean Research $26(3): 73-83$ 